\title{
A Watermark Approach for Image Transmission: Implementation of Channel Coding Technique with Security
}

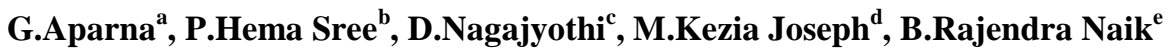 \\ a a Research Scholar, Department of Electronics and Communication Engineering, University College of Engineering, Osmania \\ University-500040, Telangana, India \\ ${ }^{\mathrm{b}}$ Associate Professor, ECE Department, CVR Engineering College, Ibrahimpatnam, Telangana \\ ${ }^{\mathbf{c}}$ Associate professor, ECE DEPARTMENT,Vardhaman College of EngineeringKacharamShamshabadHyderabadTelangana \\ ${ }^{\mathrm{d}}$ Professor, ECE Department, Stanley College of Engineering and Technology for Women, Abids, Nampally, Telangana \\ ${ }^{\mathrm{e}} \mathrm{HoD}$, ECE Department, University College of Engineering, Osmania University, Hyderabad, Telangana
}

Article History: Received: 10 November 2020; Revised 12 January 2021 Accepted: 27 January 2021; Published online: 5 April 2021

\begin{abstract}
In this paper an approach for secured digital image transmission with watermark is being proposed. The tremendous growth in technology for various applications demand secured communications across the wireless channels. Secured image transmission is the one of the prominent process in digital communication applications. A watermark is embedded in to the image data that is to be protected from unauthorized users. The cryptographic algorithms chosen for secured transmission led to the need for hardware implementation. In the process of secured image transmission turbo encoder is proposed for error correction. The proposed approach is realized in terms of hardware for the digital logic size, area and power consumption using Xilinx 14.2 software. Synthesizing and implementation of verilog code on the target device xc6slx150-2fgg 484 for timing constraints, device utilization and performance details.
\end{abstract}

(c) 2020 Elsevier Ltd. All rights reserved.

Selection and/or Peer-review under responsibility of International Conference on Mechanical, Electronics and Computer Engineering

Keywords: Encryption, Digital Image Water marking, turbo coding, JPEG 2000

\section{Introduction}

The rapid growth in wireless communications and multimedia applications has given good scope for digital image transmission with security. The implementation of the secured algorithms to ensure secured data transmission need hard ware realization in logic size, area and power consumption point of view. Research on secured transmission is focused as the information that is being transmitted through wireless channel is prone to noise mostly for which reason the data gets corrupted. Hence, turbo coded method suggested in the approach can recover the lost or errant data from the received data. The high bit error rates that are caused due to transmission of data through wireless channel can also be overcome with this approach [1]. One of the best solutions to obtain the lost is to incorporate the system with source channel coding. Turbo channel coding with iterative soft decoding provides the better performance which is nearer to optimal Shannon capacity error criteria. In today's advanced multi-media era, the size of the file (Image file) is expected to be as low as possible while preserving the quality of it. To accomplish this JPEG 2000 image standard proves to be the best in class which is very much useful for faster transmission.

The JPEG 2K-standard is low in complexity which made it ease for hardware implementation through VLSI realization [2]. This image standard is based on discrete wavelet transforms (DWT) in coordination with arithmetic entropy coding [3].

In this paper, a digital image watermarking approach with DWT and turbo coding is proposed. This approach also includes transmission of the watermarked image with high security, in order to transit at faster rate it incorporates JPEG2K standard. Lastly it investigates to implement the entire process through VLSI realization.

This paper is organized as follows; section 1 presents the need and necessity of the work and the objectives that were framed to be implemented in the work. Section- 2 presents in details description of the proposed approach. Section 3 presents the proposed watermarking technique with turbo coding concluded with experimental results in section5. 


\section{Proposed Approach}

The proposed digital watermarking method consists of JPEG compression and turbo coding at either side of encoder and decoder. This method is meant for quantized DCT bases which consist of three main process steps namely zigzag scanning coding, run length coding and Huffman coding.

When realized in hardware terms using VLSI, zigzag scanning uses dual Random Access Memory (RAM) for faster processing. On the other hand, run length coding counts the intermediate zero in between the successive non-zero Discrete Cosine Transform (DCT) elements to reduce the data size [4]. In order to reduce the complexity of the Huffman coding a lookup table with the frequency of occurrence of particular code pattern value combinations were employed in decreasing order of probabilities which are connected with inconsistent length codes. The entire process flow is designed and implemented using Low power approaches in Verilog HDL [5].
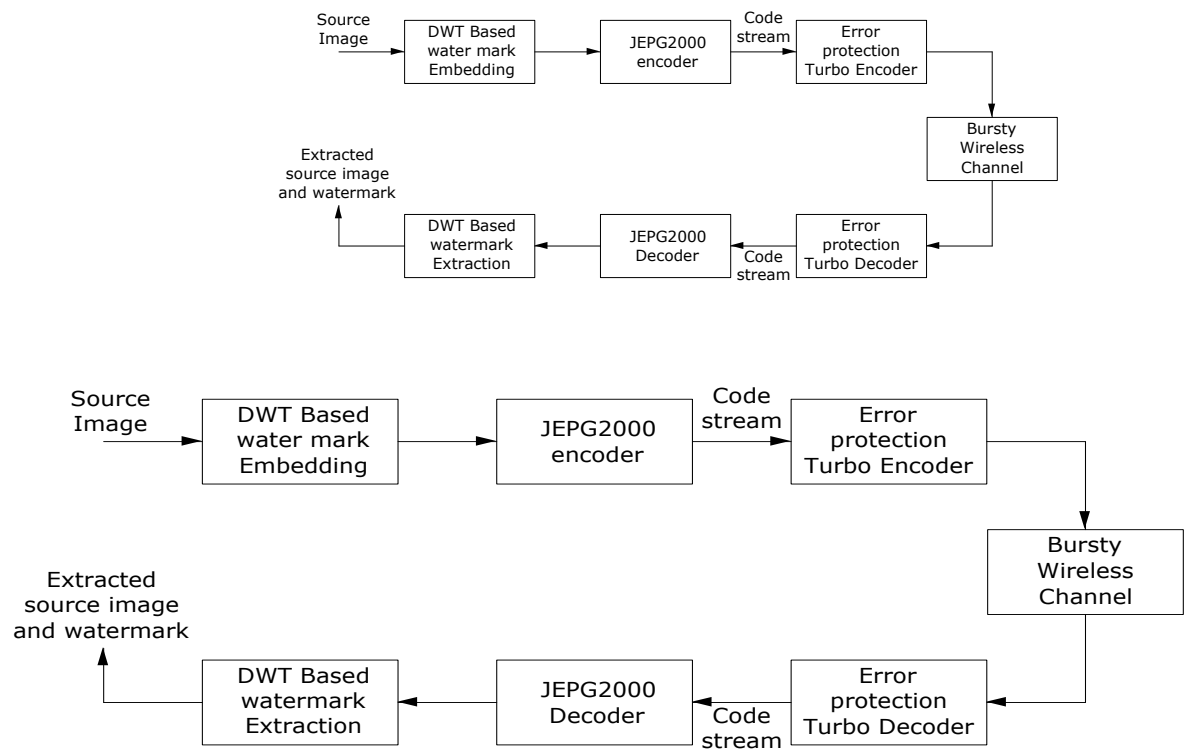

Fig. 1. Schematic block diagram of the proposed approach.

The hardware architecture for the proposed approach is synthesized with RTL compiler which is mapped into 90mm standard cells while the simulation were performed using Model Sim. The layout is designed using IC compiler. Power consumption is restricted to $0.78 \mathrm{~mW}$ for run length encoder and $0.884 \mathrm{~mW}$ for run length decoder in the stipulated area.

Below figure 1 shows the block diagram of the proposed approach of image transmission with watermarking. In this process the watermark is embedded into the cover image and then the JPEG-2K standard is performed for efficient compression. Turbo coding is employed as the channel source coding for error free transmission.

\section{Digital Water Marking Technique}

The proposed digital watermarking consists of four stages namely structuring watermark, embedding watermark, processing watermarked image and extracting the watermark. In the complete process the technique used should embed the watermark without distorting the original input image and while extracting there should not be any loss of information. The watermark should be imperceptible for few applications like secured communications in medical area especially.

The DWT and IDWT process is performed using Haar wavelet transform which can be analyzed using the following equations

$$
\begin{aligned}
& \operatorname{High}(n)=\operatorname{Im} \operatorname{age}(2 n+1)-\operatorname{Im} \operatorname{age} \ldots . . \\
& \left.\operatorname{Low}(n)=\operatorname{Im} \operatorname{age}(2 n)+\operatorname{round} \frac{\operatorname{High}(n)}{2}\right) \ldots
\end{aligned}
$$




$$
\begin{aligned}
& \operatorname{Im} \operatorname{age}(2 n)=\operatorname{Low}(n)-\operatorname{round}\left(\frac{\operatorname{high}(n)}{2}\right) \ldots \\
& \operatorname{Im} \operatorname{age}(2 n+1)=\operatorname{High}(n)+\operatorname{Im} \operatorname{age}(2 n) \ldots
\end{aligned}
$$

In numerical analysis and functional analysis DWT is employed as it captures both frequency and location information called as location in time. The main advantage of Haar wavelet is that it helps to analyze the sudden transitions in the signal. The properties of Haar transform of no multiplication requirement and equal lengths of input and output finds wide range of applications for image compression.

The watermarking algorithm is implemented in three steps:

- At first, the secret watermark most significant bits are embedded into the least significant bits of the decomposed cover image low frequency sub band using DWT and bit plane slicing as shown in below figure2.

- The watermarked image is subjected to different attacks to test its robustness and legibility over the transmission.

- At the extraction, the watermark is extracted blindly without the information of original image.

The process of embedding is replicated for both $\mathrm{LH}$ and HL components. The inverse transform is applied to the modified coefficients to obtain the watermarked spatial elements forming a watermarked image. In this approach $\mathrm{HH}$ and LL sub bands are not utilized as it was realized that they are not so robust and it find cumbersome at the extraction.

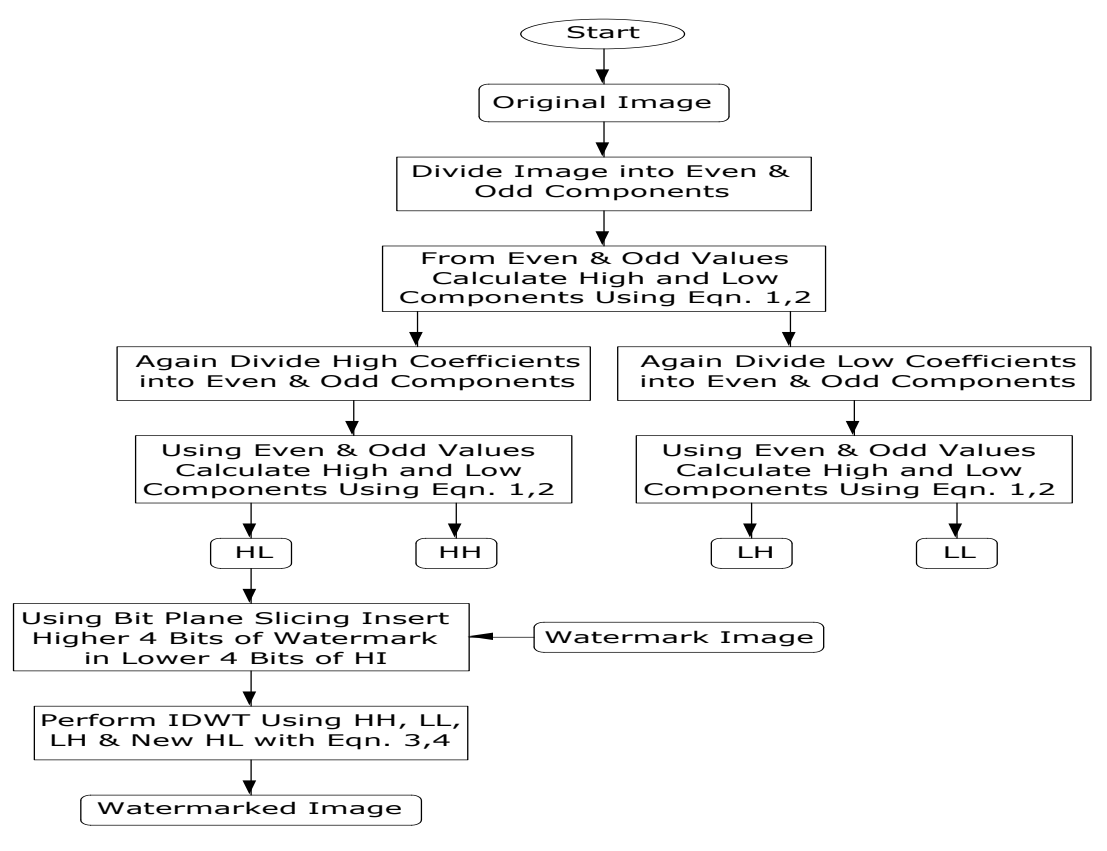

Fig. 2. flow diagram of watermarked image, Original Watermark, Extracted Watermark.

\section{Turbo Coding}

The encoder used in this approach represented in Figure 3 consists of four states convolutional encoder and an interleaver. A coder rate of $1 / 3$ turbo coding with parallel concatenation is considered however the encoder is comprised of two $1 / 2$ code rate convolutional encoders. The encoder receives un-coded data bits which processed as set of parity bits at the output. The convolutional encoder receives an interleaved sequence of these information bits. The decoding is performed using the BCJR algorithm. The main intension of the decoding algorithm is to iterate among two SISO (Soft Input / Soft Output). The decoder shown in Figure 4 receives a real value as the input signal and then outputs data information as an approximation of expressing in terms of the transmitted bits probability. In the entire design the choice of interleaver is very crucial which is meant to minimize the correlation of neighboring bits at the input of the convolutional encoder. 


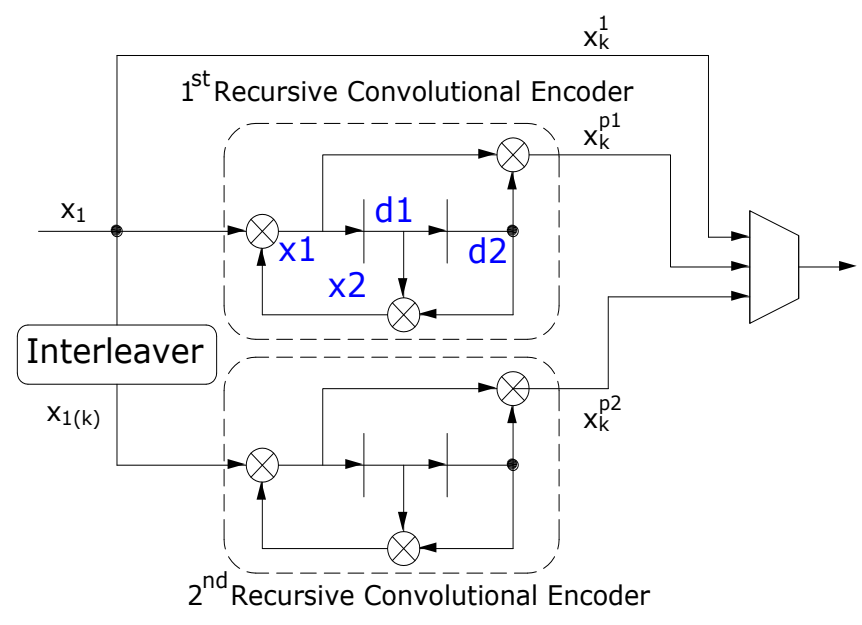

Fig. 3. The architecture of the Turbo Encoder.

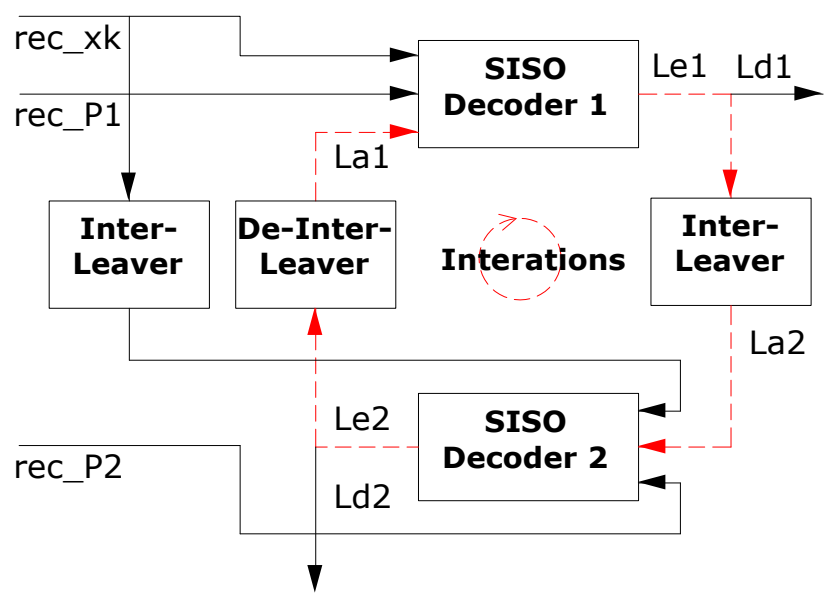

Fig. 4.The architecture of the Turbo Decoder

\section{Results}

The hardware implementation of the approach is shown in the results below in the tabular form Table 1 and Table 2 in FPGA device utilization summary of the encoder and decoder implemented on the target device. The synthesis report gives the structure of the encoder and decoder and their RTL respectively.

\begin{tabular}{|c|c|c|c|}
\hline Slice Logic Utilization & Used & Available & Utilization \\
\hline Number of Slice Registers & 1,526 & 184,304 & $1 \%$ \\
\hline Number used as Flip Flops & 1,526 & & \\
\hline Number used as Latches & 0 & & \\
\hline Number used as Latch-thrus & 0 & & \\
\hline Number used as AND/OR logics & 0 & & \\
\hline Number of Slice LUTs & 1,033 & 92,152 & $1 \%$ \\
\hline Number used as logic & 881 & 92,152 & $1 \%$ \\
\hline Number using O6 output only & 460 & & \\
\hline Number using O5 output only & 54 & & \\
\hline Number using $\mathrm{O} 5$ and $\mathrm{O} 6$ & 367 & & \\
\hline Number used as ROM & 0 & & \\
\hline Number used as Memory & 4 & 21,680 & $1 \%$ \\
\hline
\end{tabular}


Number used as Dual Port RAM 0

Number used as Single Port RAM 0

Number used as Shift Register 4

Number using O6 output only 4

Number using O5 output only 0

Number using O5 and O6 0

Number used exclusively as route- 148

thrus

Number with same-slice register load 142

Number with same-slice carry load 6

Number with other load 0

Number of occupied Slices

Nummber of MUXCYs used 204

Number of LUT Flip Flop pairs used $\quad 1,451$

Number with an unused Flip Flop $\quad 217$

Number with an unused LUT 418

Number of fully used LUT-FF pairs $\quad 816$

Number of unique control sets $\quad 69$

Number of slice register sites 198

lost to control set restrictions

Number of bonded IOBs 207

Number of RAMB16BWERs 0

Number of RAMB8BWERs 10

Number of BUFIO2/BUFIO2_2CLKs 0

Number

of 0

BUFIO2FB/BUFIO2FB_2CLKs

Number of BUFG/BUFGMUXs

Number used as BUFGs

Number used as BUFGMUX

Number of ILOGIC2/ISERDES2s 0

Number

of 0

IODELAY2/IODRP2/IODRP2 MCBs

Number of OLOGIC2/OSERDES2s 0

Number of BSCANs 0

Number of BUFHs 0

Number of BUFPLLs

Number of BUFPLL_MCBs 0

Number of DSP48A1s 0

Number of ICAPs 0

Number of MCBs 0

Number of PCILOGICSEs 0

Number of PLL_ADVs 0

Number of PMVs 0

Number of STARTUPs 0

Number of SUSPEND_SYNCs 0

Average Fanout of Non-Clock Nets $\quad 3.26$

$23,038 \quad 1 \%$

$46,076 \quad 1 \%$

$1,451 \quad 14 \%$

$1,451 \quad 28 \%$

$1,451 \quad 56 \%$

$184,304 \quad 1 \%$

$338 \quad 61 \%$

$268 \quad 0 \%$

$536 \quad 1 \%$

$32 \quad 0 \%$

$32 \quad 0 \%$

$16 \quad 6 \%$

$12 \quad 0 \%$

$586 \quad 0 \%$

$586 \quad 0 \%$

$586 \quad 0 \%$

$4 \quad 0 \%$

$384 \quad 0 \%$

$8 \quad 0 \%$

$4 \quad 0 \%$

$180 \quad 0 \%$

$1 \quad 0 \%$

$4 \quad 0 \%$

$20 \%$

$6 \quad 0 \%$

$1 \quad 0 \%$

$1 \quad 0 \%$

$10 \%$

Table 2. FPGA Device Utilization summary

\begin{tabular}{llll}
\hline Slice Logic Utilization & Used & Available & Utilization \\
\hline Number of Slice Registers & 1,198 & 184,304 & $1 \%$ \\
Number used as Flip Flops & 1,198 & & \\
Number used as Latches & 0 & & \\
Number used as Latch-thrus & 0 & & \\
Number used as AND/OR logics & 0 & & \\
Number of Slice LUTs & 968 & 92,152 & $1 \%$ \\
Number used as logic & 827 & 92,152 & \\
Number using O6 output only & 572 & & \\
Number using O5 output only & 63 & & \\
Number using O5 and O6 & 192 & & \\
Number used as ROM & 0 & &
\end{tabular}




\begin{tabular}{|c|c|c|c|}
\hline Number used as Memory & 0 & 21,680 & $0 \%$ \\
\hline $\begin{array}{l}\text { Number used exclusively as route- } \\
\text { thrus }\end{array}$ & 141 & & \\
\hline $\begin{array}{l}\text { Number with same-slice register } \\
\text { load }\end{array}$ & 132 & & \\
\hline Number with same-slice carry load & 9 & & \\
\hline Number with other load & 0 & & \\
\hline Number of occupied Slices & 403 & 23,038 & $1 \%$ \\
\hline Number of MUXCYs used & 168 & 46,076 & $1 \%$ \\
\hline Number of LUT Flip Flop pairs used & 1,233 & & \\
\hline Number with an unused Flip Flop & 272 & 1,233 & $22 \%$ \\
\hline Number with an unused LUT & 265 & 1,233 & $21 \%$ \\
\hline Number of fully used LUT-FF pairs & 696 & 1,233 & $56 \%$ \\
\hline Number of unique control sets & 69 & & \\
\hline $\begin{array}{l}\text { Number of slice register sites } \\
\text { lost to control set restrictions }\end{array}$ & 186 & 184,304 & $1 \%$ \\
\hline Number of bondedIOBs & 86 & 338 & $25 \%$ \\
\hline Number of RAMB $\overline{16 B W E R s}$ & 0 & 268 & $0 \%$ \\
\hline Number of RAMB8BWERs & 10 & 536 & $1 \%$ \\
\hline Number of BUFIO2/BUFIO2_2CLKs & 0 & 32 & $0 \%$ \\
\hline $\begin{array}{l}\text { Number } \\
\text { BUFIO2FB/BUFIO2FB_2CLKs }\end{array}$ & 0 & 32 & $0 \%$ \\
\hline Number of BUFG/BUFGMUXs & 2 & 16 & $12 \%$ \\
\hline Number used as BUFGs & 2 & & \\
\hline Number used as BUFGMUX & 0 & & \\
\hline Number of DCM/DCM_CLKGENs & 0 & 12 & $0 \%$ \\
\hline Number of ILOGIC2/ISERDES2s & 0 & 586 & $0 \%$ \\
\hline $\begin{array}{l}\text { Number } \\
\text { IODELAY2/IODRP2/IODRP2_MCBs }\end{array}$ & 0 & 586 & $0 \%$ \\
\hline Number of OLOGIC2/OSERDES2s & 0 & 586 & $0 \%$ \\
\hline Number of BSCANs & 0 & 4 & $0 \%$ \\
\hline Number of BUFHs & 0 & 384 & $0 \%$ \\
\hline Number of BUFPLLs & 0 & 8 & $0 \%$ \\
\hline Number of BUFPLL_MCBs & 0 & 4 & $0 \%$ \\
\hline Number of DSP48A1s & 0 & 180 & $0 \%$ \\
\hline Number of ICAPs & 0 & 1 & $0 \%$ \\
\hline Number of MCBs & 0 & 4 & $0 \%$ \\
\hline Number of PCILOGICSEs & 0 & 2 & $0 \%$ \\
\hline Number of PLL_ADVs & 0 & 6 & $0 \%$ \\
\hline Number of PMVs & 0 & 1 & $0 \%$ \\
\hline Number of STARTUPs & 0 & 1 & $0 \%$ \\
\hline Number of SUSPEND_SYNCs & 0 & 1 & $0 \%$ \\
\hline Average Fanout of Non-Clock Nets & 3.38 & & \\
\hline
\end{tabular}

The Xilinx ISE (Integrated Software Environment) is used to perform synthesis. The synthesis report of encoder and decoder, the simulation output, the power report of the encoder and decoder, the timing report of the encoder and decoder, the delay report of encoder and decoder and the area report of the encoder and decoder, the RTL view of encoder and decoder are shown in the various figure 5,6,7,8 below. 


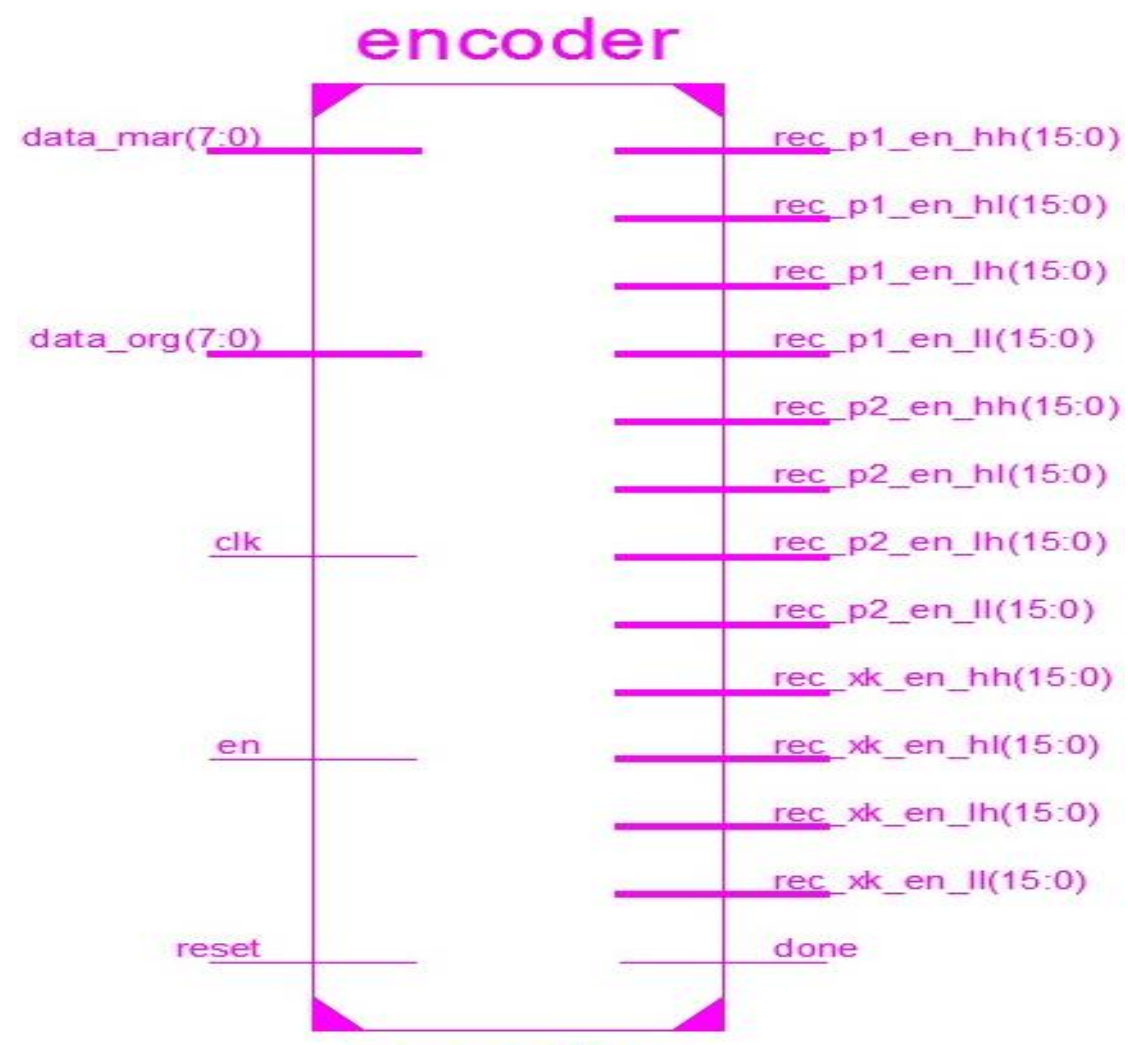

encoder

Figure 5 Encoder

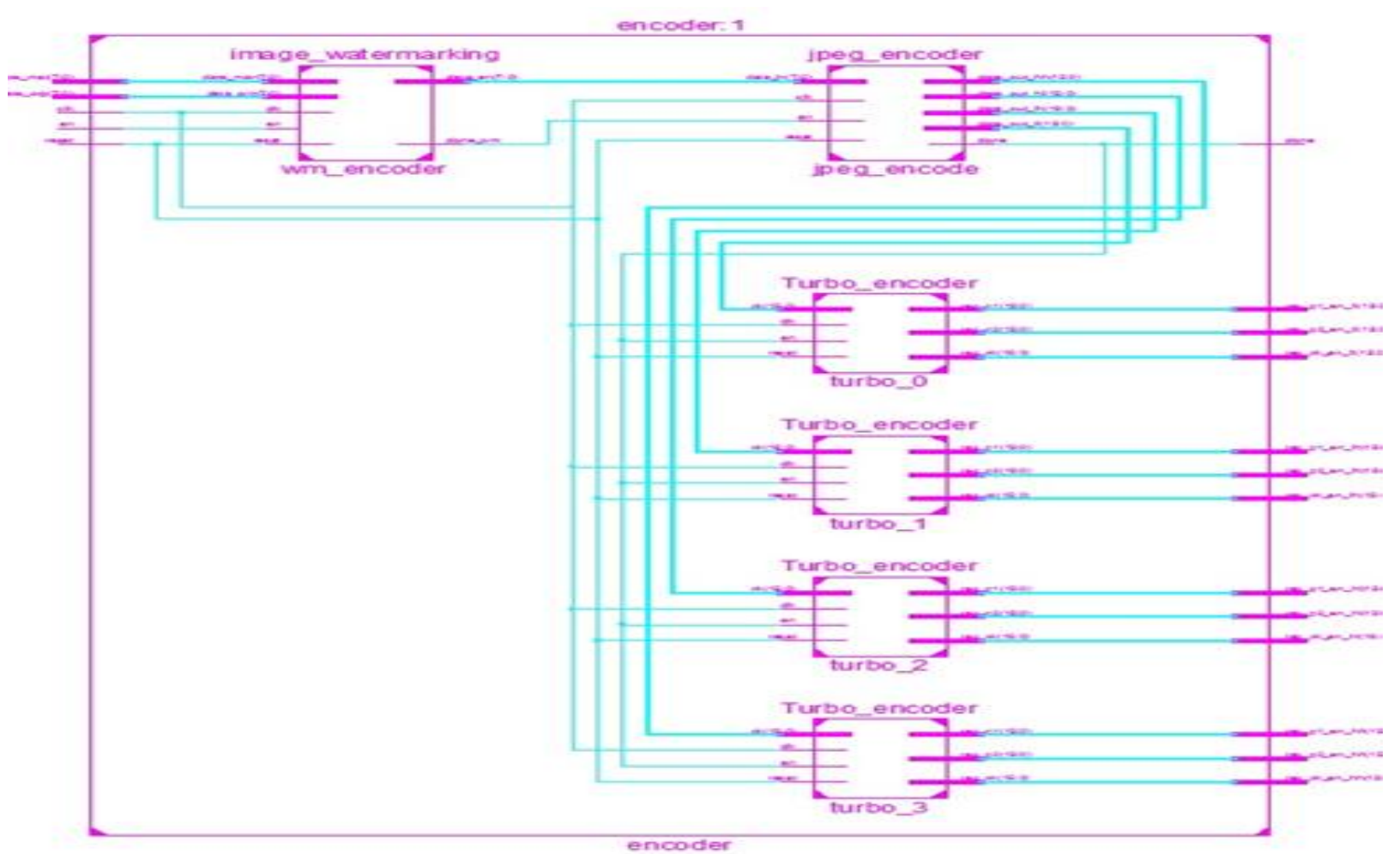

Fig.6 .RTL view of Encoder 
Numerical Values of few parameters observed from the results. FPGA used for implementing is Xilinx Spartan6 XC6slx150-2fgg484.

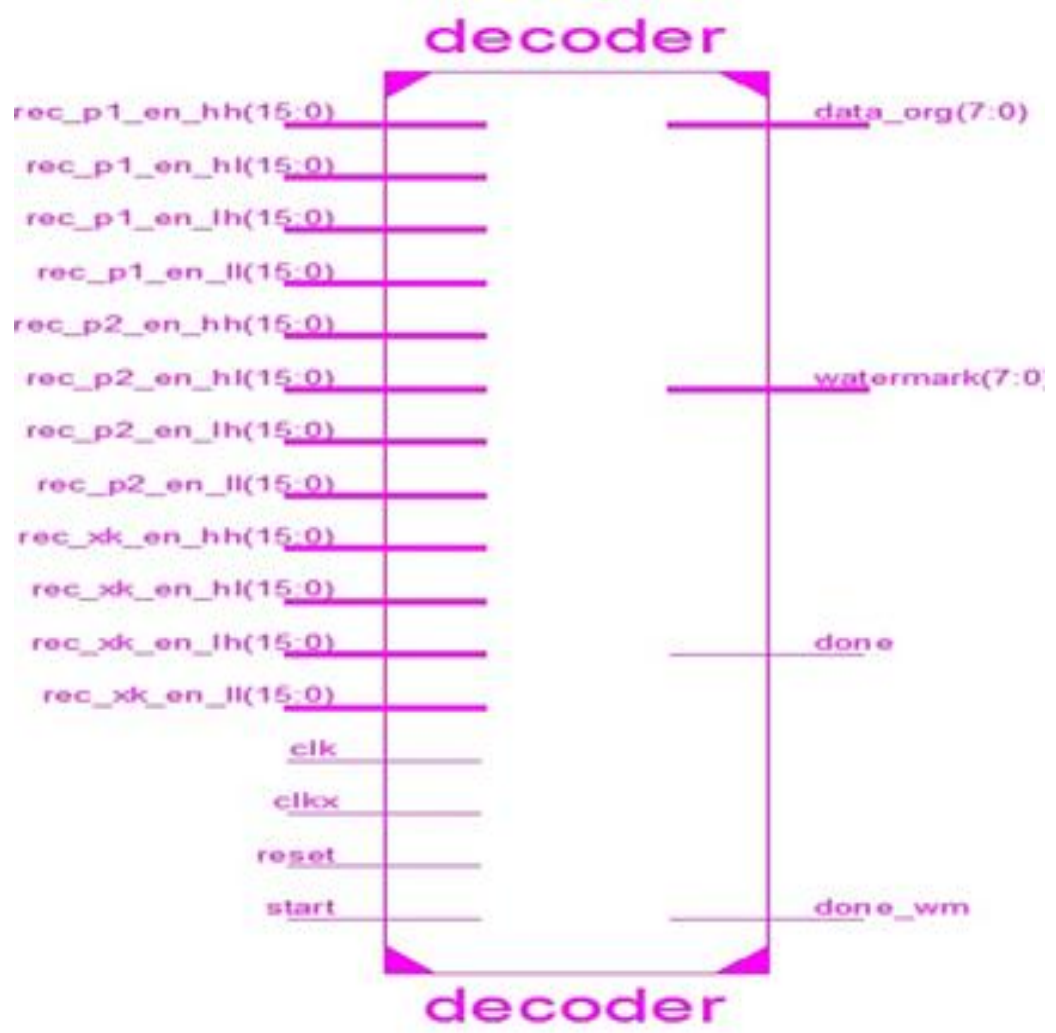

Figure 7 decoder

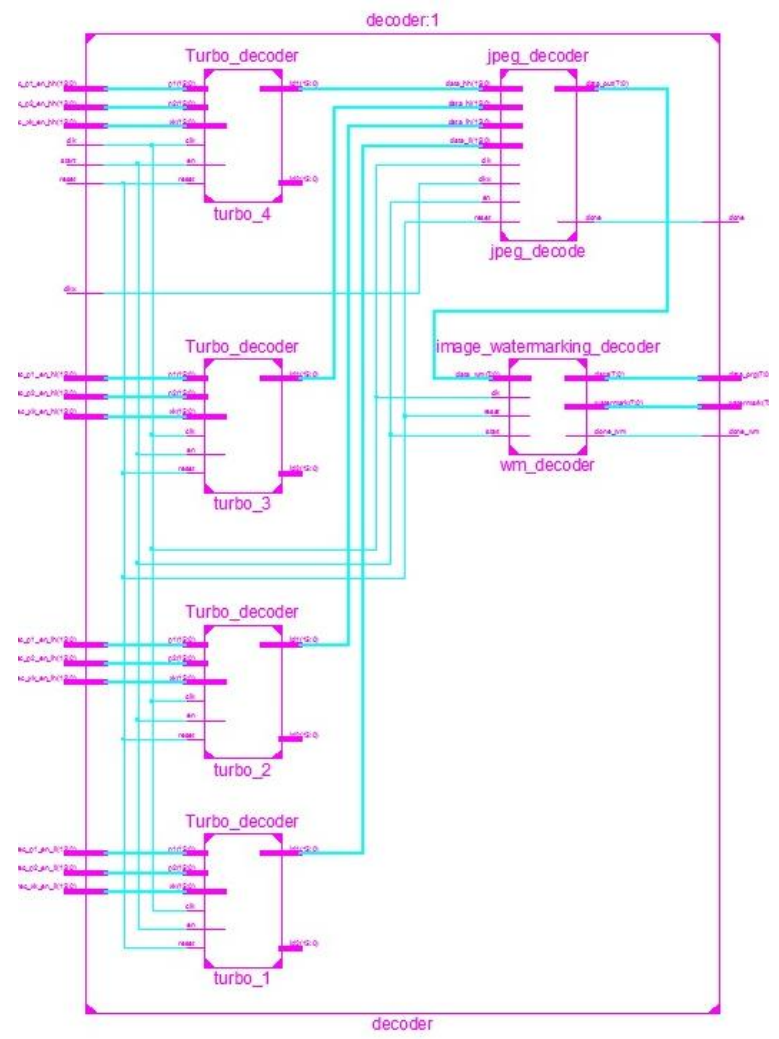

Fig. 8. RTL view of Decoder 


\section{Conclusions}

In this paper an approach for image transmission with security based on Haar Wavelet, JPEG compression and turbo decoder is realized. Results illustrate the hardware realization of the secured transmission approach. From the results it is accomplished that the proposed watermarked approach is efficient and robust as well for digital image transmission with security over wireless channels

\section{References}

Rao, K. D. (2010, October). New approach for digital image watermarking and transmission over bursty wireless channels. In IEEE 10th INTERNATIONAL CONFERENCE ON SIGNAL PROCESSING PROCEEDINGS (pp. 1829-1832). IEEE.

Antonini, M., Barlaud, M., Mathieu, P., \& Daubechies, I. (1992). Image coding using wavelet transform. IEEE Transactions on image processing, 1(2), 205-220.

Gonzalez, R. C., Woods, R. E., \& Eddins, S. L. (2004). Digital image processing using MATLAB. Pearson Education India.

Christopoulos, C., Skodras, A., \& Ebrahimi, T. (2000). The JPEG2000 still image coding system: an overview. IEEE transactions on consumer electronics, 46(4), 1103-1127.

Palnitkar, S. (2003). Verilog HDL: a guide to digital design and synthesis (Vol. 1). Prentice Hall Professional.

I. Cox, J. Kilian, T. Leighton and T. Shamoon, "Secure spread spectrum watermarking for multimedia". IEEE Transaction on Image processing,Vol 6, issue 12, pp1673-1687, 1997.

Jiri Fridrich ."A New Steganographyic Method for Palette-Based Images". Center for Intelligent Systems, SUNYBinghamton, Binghamton, NY 13902-6000. U.S Government, a grant number F30602-98-c-0009.

M.Kutter, E. Jordan, and E. Bossen ; "Digital signature of Color images using amplitude modulation", J. Electron Imaging, vol. 7, (2), pp.326-332, 1998.

E.T. Lin, E.J. Delp. "A review of data hiding in images", Proceedings of the conference on image process image quality image capture systems, PICS'99'. 25-28, April 1999, savannah, Georgia, pp. 274-278

BENDER, W. GRUHL, D. MORIMOTO N, and A. LU, "Techniques for data Hiding”, IBM, syst. J., 35, (3\&4) pp.313-336, 1996.

S. K. Moon and R.S. Kawitkar, "Data Security using Data Hiding", IEEE International conference on computational intelligence and multimedia applications, vol. 4, pp. 247-251, Dec. 2007.

KO-Chin Chang, Chien-Ping Chang, Ping S.Huang, and Te-mingTu.:"A novel image steganographic method using Tri-way pixel value Differencing".Journal of multimedia, Vol.3, No.2, June-2008.

K.Suresh Babu, K. B. Raja, Kiran Kumar k, Manjula Devi T H, Venugopal K R ,L.M Patnaik. "Authentication of secret information in image steganography;"TENCON-2008, IEEE Region 10 Conference. pp. 1-6, Nov 2008. 